\title{
Magnesium Sulfate: A Dual-Scattering Unconventional Negative Stain
}

\author{
W. H. Massover
}

Department of Cell Biology and Molecular Medicine, UMDNJ- New Jersey Medical School, Newark, NJ 07101-1709

All common negative stains are salts of heavy metals where only one ion contains a strongly scattering element. Accordingly, these often are categorized as being either an anionic or a cationic negative stain [e.g., 1]. Although a very high contrast for imaging weakly scattering protein molecules and assemblies is provided, heavy metal negative stains are missing many other properties essential for structural studies at high resolution (e.g., small probe size, physiological $\mathrm{pH}$ and composition, maintenance of native structure during drying, resistance to beam-induced movements) [2]. These deficiencies result in the usual image resolution being limited to the quaternary level of protein structure (i.e., $25 \AA(2.5 \mathrm{~nm})$ ). Recognition that many light elements provide enough scattering to give the appositional contrast required for negative staining considerably enlarges the choice of stain agents [3]. The properties missing for higher resolution negative staining are such that it is very unlikely that any one new compound will be able to satisfy all the defects; rather, it seems likely that a complex mixture of compounds, each having one or another specific desired benefit, will be required [4]. The present study evaluates the ability of Epsom salt, magnesium sulfate, to function as an experimental negative stain, and examines whether the properties of this divalent metal salt will be useful for inclusion in negative stain cocktails capable of revealing higher resolution protein structure.

Thin 3-D crystals of bovine liver catalase [5] are used as test specimens. Suspended crystals are deposited onto a glow-discharge-treated thin carbon support film overlying a 400-mesh grid, drained and exposed to negative stain, re-drained, and then rapidly dried [2-4]. All stain solutions include $150 \AA$ protein-A-coated colloidal gold (Ted Pella, Inc.) as a focusing aid. Specimens are examined at $100 \mathrm{kV}$ [2,3]; low-dose images are recorded with a Tecnai-12 electron microscope (FEI Co.) using the Philips computer-controlled system with a 4096x4096 CCD camera (Ultrascan: Gatan, Inc.).

Magnesium sulfate $\left(\mathrm{MgSO}_{4} \cdot 7 \mathrm{H}_{2} \mathrm{O} ; \mathrm{F} . \mathrm{W} .=246.5\right)$ at $200 \mathrm{mM}$ has a natural $\mathrm{pH}$ of 5.7 and remains fully soluble when neutralized to pH7.0. It mostly dries into a thin layer giving a diffuse ring pattern with electron diffraction, indicating a glassy state. The periodic protein lattice in orthorhombic catalase crystals [5] is readily imaged with exposure to $100-200 \mathrm{mM}$ magnesium sulfate (Fig. 1). The image contrast level can approach that obtained with the conventional heavy metal negative stain, ammonium molybdate (1-2\%). Low-dose images thus far have not yielded Fourier transform spots at higher resolution levels; however, this result is consistent with the postulated requirement for use of mixtures rather than single stain agents by themselves. A bubbling response to the electron beam irradiation is detected only in thick regions of this dried stain.

The results clearly establish that magnesium sulfate functions successfully as a negative stain. This light-atom salt provides notably more image contrast than magnesium acetate [6]; it is likely that this increase is due to the fact that strongly scattering atoms are contained in both the anion (S) and the cation $(\mathrm{Mg})$ of the former (i.e., dual-scattering), while the acetate salt only has significant scattering by its cation (i.e., mono-scattering). Thus, magnesium sulfate simultaneously acts as both an anionic and a cationic negative stain. Magnesium sulfate also has been utilized by biochemists to enhance 
retention of native protein structure in isolated polypeptides, and is used in some mother liquors for generating protein crystals suitable for x-ray diffraction studies. These several beneficial optical and non-optical properties of magnesium sulfate encourage the expectation that it will be useful as a component of new negative stain cocktails designed to improve the imaging of native protein structure at higher resolution levels [7].

\section{References}

[1] T.S. Baker et al., J. Mol. Biol. 184 (1985) 81-98.

[2] W.H. Massover and P. Marsh, Ultramicrosc. 85 (2000) 107-121.

[3] W.H. Massover and P. Marsh, Ultramicrosc. 69 (1997) 139-150.

[4] W.H. Massover, Proc. 13 ${ }^{\text {th }}$. Europ. Microsc. Cong. III (2004) 417-418.

[5] P.N.T. Unwin, J. Mol. Biol. 98 (1975) 235-242.

[6] W.H. Massover, Microsc. Microanal. 6 suppl. 2 (2000) 478-479.

[7] This research is supported in part by private funds.

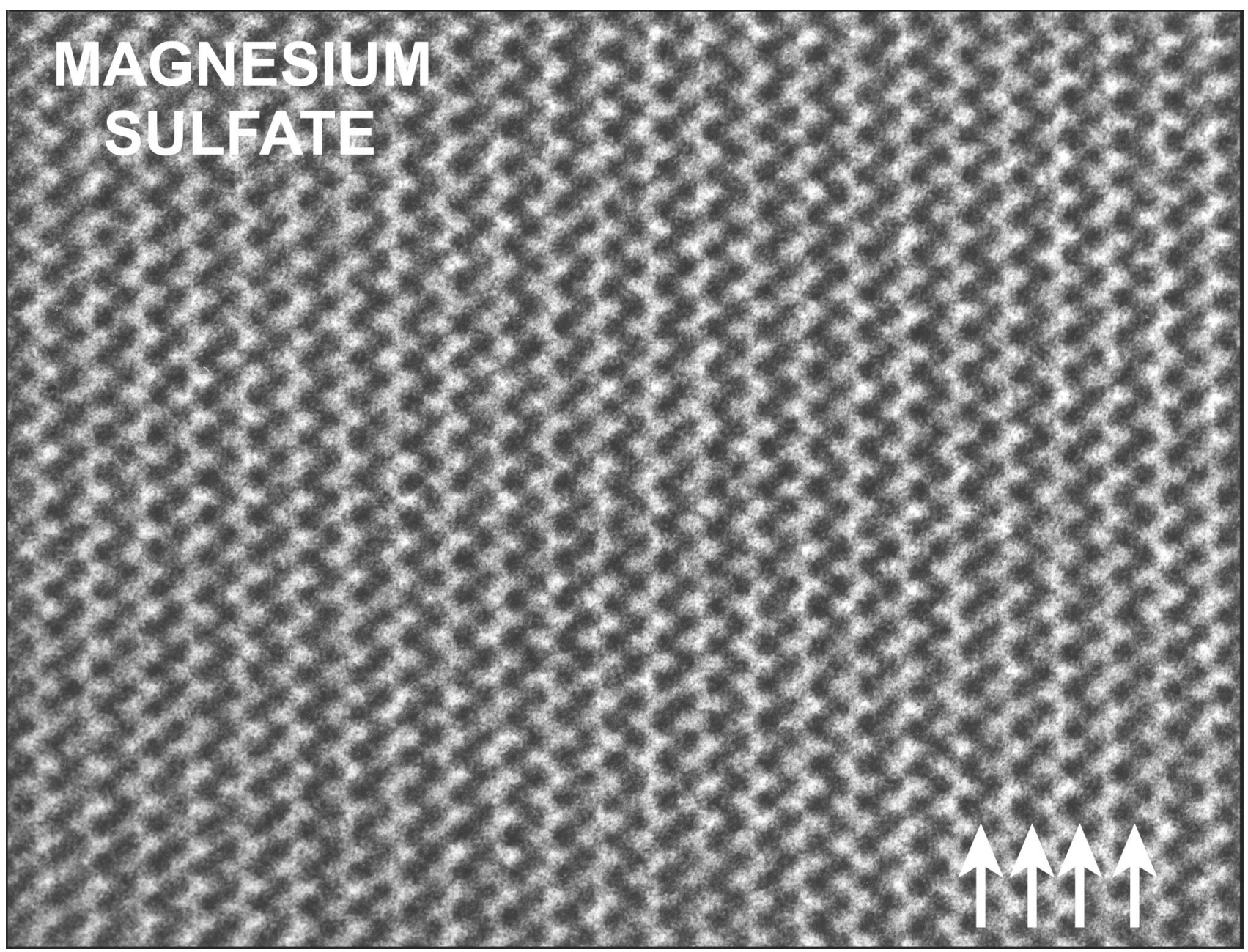

FIG. 1. Thin catalase crystal negatively stained with $200 \mathrm{mM}$ magnesium sulfate. Protein is light; stain is dark. In addition to the major $87 \AA$ half-periods in the orthorhombic lattice (arrows), smaller periodicities can be discerned by viewing at a lower angle along several different orientations. 